\title{
Relação entre porosidade e os módulos elásticos de esponjas cerâmicas produzidas via "gelcasting"
}

\section{(The relation between porosity and elastic moduli of gelcast ceramic foams)}

\author{
J. A. Rodrigues', F. S. Ortega ${ }^{2}$, A. E. M. Paiva ${ }^{3}$, E. L. G. Villaboim ${ }^{1}$, V. C. Pandolfelli ${ }^{1}$ \\ ${ }^{l}$ Grupo de Engenharia de Microestrutura de Materiais, DEMa, UFSCar \\ Rod. Washington Luiz, km 235, C.P. 676, S. Carlos, SP 13565-905 \\ josear@power.ufscar.br,vicpando@power.ufscar.br \\ ${ }^{2}$ UNIVAP, S. José dos Campos, SP \\ ${ }^{3}$ Centro Federal de Educação Tecnológica do Maranhão
}

\begin{abstract}
Resumo
A técnica "gelcasting", associada à aeração de suspensões cerâmicas, permite a produção de esponjas cerâmicas com uma larga faixa de porosidade. Esponjas à base de alumina foram produzidas com porosidade variando de 62 a 87 \%-vol, o que corresponde a densidades relativas entre 0,13 e 0,38 . Essas esponjas foram utilizadas para a verificação de modelos, tais como de Mackenzie, de Boccaccini e de Gibson e Ashby, que relacionam a porosidade ou a densidade com os módulos elásticos da cerâmica porosa. Os módulos elásticos de Young, de cisalhamento e a razão de Poisson foram caracterizados através da técnica de ressonância mecânica de barras. Essa técnica permitiu avaliar os valores dos módulos elásticos mesmo com o material tendo macroestrutura celular. Os resultados permitiram verificar qual dos citados modelos oferece melhor descrição do comportamento do módulo elástico em função da porosidade das esponjas cerâmicas.

Palavras-chave: cerâmica porosa, gelcasting, módulo elástico, ressonância de barras, alumina.
\end{abstract}

Abstract

Broad porosity range can be obtained in ceramic foams employing the gelcasting technique coupled to the aeration of ceramic suspensions. Alumina based foams were produced with porosity in the range of 62 to 87 volume-\%, which corresponded to relative densities between 0.13 and 0.38. Fitting models after Mackenzie, Boccaccini and Gibson and Ashby, describing the dependency of elastic moduli on density or porosity, were applied. The bar resonance technique was used to determine the values of Young's modulus, shear modulus and Poisson's ratio of these cellular material. The fitting model that best describe the dependence of elastic moduli on porosity could be identified and discussed.

Keywords: ceramic foams, gelcasting, elastic modulus, bar resonance, alumina.

\section{INTRODUÇÃO}

Cerâmicas porosas celulares com uma ampla faixa de valores de porosidade e de propriedades mecânicas podem ser produzidas associando-se a técnica de aeração de suspensões com o processo "gelcasting" [1]. Entretanto, o uso destes materiais em aplicações específicas como, as estruturais, isolamento térmico, filtragem, suportes para catalisadores, imobilização de microorganismos em aplicações biotecnológicas e implantes ósseos, requer uma adequação da macroestrutura do material que favoreça propriedades específicas, tais como resistência mecânica, módulo elástico aparente, permeabilidade, condutividade térmica ou área superficial [2]. Os valores dessas propriedades decorrem da porosidade, distribuição do tamanho de poros e conectividade entre células vizinhas, características estas definidas durante a produção da cerâmica.

A introdução de poros nos materiais cerâmicos causa, entretanto, a redução da resistência mecânica e do módulo elástico aparente destes materiais [3]. Além disso, a maioria dos processos de fabricação de cerâmicas porosas proporciona corpos com grande quantidade de trincas e macrodefeitos, provocando uma deterioração adicional de suas propriedades mecânicas.

Sepulveda [4] desenvolveu um novo processo para fabricação de cerâmicas porosas, baseado na aeração de uma suspensão cerâmica, contendo um agente espumante. A espuma cerâmica obtida é rapidamente gelificada através da polimerização in situ de monômeros previamente adicionados e posteriormente sinterizada. Este processo proporciona cerâmicas com valores de porosidade que podem ultrapassar $90 \%$ e uma macroestrutura celular homogênea constituída de poros com geometria aproximadamente esférica e paredes densas com orifícios que conectam células adjacentes. Estas características tendem a melhorar as propriedades mecânicas destes materiais, já que os poros são os defeitos críticos 
determinantes. Comparadas às cerâmicas feitas pelo método da réplica de esponjas poliméricas, que possuem filamentos ocos com secção triangular, aquelas produzidas por gelcasting possuem uma macroestrutura mais favorável, do ponto de vista mecânico.

Para a compreensão do comportamento mecânico em geral e em particular dos danos causados nos materiais após choque térmico, os valores dos módulos elásticos têm importância particular, já que os mesmos entram no equacionamento geral da resistência mecânica e dos parâmetros de resistência ao dano por choque térmico. Principalmente para as esponjas cerâmicas usadas como filtros para aplicações em altas temperaturas, esses aspectos são ainda mais relevantes.

Com esponjas cerâmicas de alta porosidade, nas quais as paredes das suas células constituem filamentos finos, a medida dos módulos elásticos e da razão de Poisson pelos métodos mecânicos convencionais de tração, compressão ou flexão, é extremamente problemática. Por isso, o emprego de técnicas de ressonância mecânica de barras [5-7], com as quais se pode aplicar baixas cargas ao corpo de prova, é de grande importância.

Neste trabalho aplicou-se a técnica de ressonância mecânica de barras para a caracterização do módulo de Young, do módulo de cisalhamento e da razão de Poisson, de esponjas de alumina produzidas por aeração/gelcasting, com faixa de porosidade entre 62 e $87 \%$-vol., correspondente a densidades relativas entre 0,13 e 0,38 .

\section{Ressonância mecânica de barras}

A teoria básica para as medidas das propriedades elásticas através do método de ressonância mecânica de barras é descrita por diversos autores [8-11]. Para um corpo de prova prismático de secção transversal retangular e dimensões $\mathbf{L}$ de comprimento, $\mathbf{b}$ de largura e a de espessura, com freqüência de ressonância torcional $\mathbf{f}_{\text {tn }}$, para o $\mathbf{n}$-ésimo modo de vibração, e ainda com densidade aparente $\rho$, o módulo de cisalhamento, $\mathbf{G}$, é dado por $[10,11]$ :

$$
G=\rho\left(\frac{2 \cdot L \cdot f_{t n}}{n}\right)^{2} \cdot R_{n}
$$

$R_{n}=R_{0} \cdot\left[1+n^{2}\left(\frac{b}{L}\right)^{2}\left(0,01746+0,00148 n+0,00009 n^{2}\right)\right](\mathrm{B})$

onde $\mathbf{R}_{\mathbf{0}}$ é uma constante dependente de $\mathbf{a}$ e $\mathbf{b}$ e escrita como

$$
R_{0}=\frac{1+\left(\frac{b}{a}\right)^{2}}{4-2,521 \cdot\left(\frac{a}{b}\right) \cdot\left[1-\frac{1,991}{\left.e^{\pi \cdot\left(\frac{b}{a}\right)}+1\right]}\right]}
$$

Para a mesma barra, vibrando em flexão, o módulo de Young, E, pode ser obtido pela equação $[8,9]$ :

$$
E=\frac{4 \cdot \pi^{2} \cdot f_{f n}^{2} \cdot L^{4} \cdot \rho}{m_{n}^{4} \cdot \frac{a^{2}}{12}}
$$

onde $\mathbf{f}_{\mathbf{f n}}$ representa as freqüências flexionais de ressonância, $\mathbf{m}_{\mathbf{n}}$ são raízes de funções do tipo $\mathbf{g}_{\mathbf{n}}\left(\mathbf{m}_{\mathbf{n}}, \mu, \mathbf{a}, \mathbf{L}\right)=\mathbf{0}$ que, além das dimensões do corpo de prova, dependem também da razão de Poisson, $\mu$.

Quando a razão de Poisson não é conhecida previamente, deve-se proceder a um cálculo iterativo [12] a partir de um valor inicial de $\mu$. Nesse caso, deve-se também utilizar a relação entre os módulos elásticos e $\mu$, válida para materiais isotrópicos, dada por:

$$
\mu=\frac{E}{2 G}-1
$$

\section{Dependência com a porosidade}

São vários os modelos que descrevem a dependência dos módulos elásticos com a porosidade ou com a densidade relativa de um corpo sólido.

Na hipótese de que um material contém poros com simetria esferoidal, Asmani et al. [13] mostram, utilizando uma equação de Boccaccini [14], derivada de um trabalho de Ondracek [15], que a dependência do módulo de Young aparente, E, com a porosidade total, $\mathbf{P}$, pode ser escrita como segue:

$$
E(P)=E_{0} \cdot\left(1-P^{2 / 3}\right)^{s}
$$

onde $\mathbf{E}_{\mathbf{0}}$ é o módulo de Young para o material denso $\left(\mathbf{E}_{\mathbf{0}}=400\right.$ GPa para alumina densa [16]) e o expoente s é calculado através de:

$$
s=1,21 \cdot\left(\frac{z}{x}\right)^{\frac{1}{3}} \cdot\left\{1+\left[\left(\frac{z}{x}\right)^{-2}-1\right] \cdot \cos ^{2}(\phi)\right\}^{\frac{1}{2}}
$$

onde $\mathbf{z} / \mathbf{x}$ é a razão entre os eixos principais dos poros (fator de forma) e $\cos ^{2}(\phi)$ é um fator de orientação desses poros (para orientação aleatória $\cos ^{2}(\phi)=1 / 3$ ). Para poros esféricos, $\mathbf{z} / \mathbf{x}=$ $1, \mathbf{s}$ assume o valor 1,21 e para poros alongados, por exemplo, $\operatorname{com} \mathbf{z} / \mathbf{x}=1,5$, s seria igual a 1,25 .

Dörre e Hübner [16] mostram, segundo o modelo de Mackenzie [17], que se baseia na concentração de tensão nos poros, que para um corpo isotrópico e homogêneo, contendo um grande número de vazios esféricos e distribuídos aleatoriamente, a dependência do módulo de Young com a porosidade total pode ser descrita por: 


$$
E(P)=E_{0} \cdot\left(1-k \cdot P+(k-1) \cdot P^{2}\right)
$$

onde $\mathbf{k}$ é uma constante definida em função dos valores do módulo de cisalhamento, $\mathbf{G}_{\mathbf{0}}$, e do módulo volumétrico, $\mathbf{K}_{\mathbf{0}}$, ambos do material denso, segundo a expressão:

$$
k=\frac{5\left(3 K_{0}+4 G_{0}\right)}{9 K_{0}+8 G_{0}}
$$

Já que se assume a homogeneidade e a isotropia do material, as relações entre os módulos elásticos e a razão de Poisson, podem ser empregadas, ou seja,

$$
K_{0}=\frac{E_{0}}{3\left(1-2 \mu_{0}\right)}
$$

e

$$
G_{0}=\frac{E_{0}}{2\left(1+\mu_{0}\right)}
$$

onde $\mu_{0}$ é a razão de Poisson do material denso. Substituindose as equações $\mathbf{J}$ e $\mathrm{K}$ em $\mathrm{I}$, tem-se a expressão de $\mathbf{k}$ como função somente da razão de Poisson, como segue:

$$
k=\frac{15}{7} \cdot\left(\frac{1-\mu_{0}}{1-\frac{5}{7} \cdot \mu_{0}}\right)
$$

Na Tabela I mostram-se alguns valores de $\mathbf{k}$ para diferentes valores da razão de Poisson.

Tabela I - Alguns valores de $\mathbf{k}$ para diferentes valores de $\mu_{0}$. [Table I - Some values of $\boldsymbol{k}$ for some different values of $\mu_{0}$.]

\begin{tabular}{cc}
\hline$\mu_{\mathbf{0}}$ & $\mathbf{k}$ \\
\hline 0,20 & 2,00 \\
\hline 0,25 & 1,96 \\
\hline 0,31 & 1,90 \\
\hline
\end{tabular}

Nota-se, portanto, que ao se assumir o valor de $\mu_{0}=0,20$, k fica igual a 2, o que transforma a equação $\mathrm{H}$ em:

$$
E(P)=E_{0} \cdot\left(1-2 \cdot P+P^{2}\right)
$$

Considerando-se que arelação entre a porosidade total e a densidade relativa, $\rho / \rho_{0}$, do material poroso pode ser escrita como [18]:

$$
P=1-\frac{\rho}{\rho_{0}}
$$

onde $\rho$ e $\rho_{0}$ são as massas específicas (densidade) do material poroso e do material denso, respectivamente, a substituição da equação $\mathrm{N}$ na $\mathrm{M}$, gera a relação

$$
E\left(\frac{\rho}{\rho_{0}}\right)=E_{0} \cdot\left(\frac{\rho}{\rho_{0}}\right)^{2}
$$

A menos de uma constante $\mathbf{C}$, a equação $\mathrm{O}$ é a relação apresentada por Gibson e Ashby [18] entre o módulo de Young e a densidade relativa de cerâmicas celulares. A equação geral [19] para essa relação pode ser escrita como:

$$
E\left(\frac{\rho}{\rho_{0}}\right)=C \cdot E_{0} \cdot\left(\frac{\rho}{\rho_{0}}\right)^{n}
$$

onde $\mathbf{C}$ e $\mathbf{n}$ são constantes que para sólidos celulares de baixas massas específicas, dependem da microestrutura do material sólido. Os valores de $\mathbf{n}$, geralmente se encontram entre 1 e 4 . No caso de esponjas cerâmicas de células fechadas, $1<\mathbf{n}<2$. Ao nível da escala das células, os fatores que influenciam os valores de $\mathbf{C}$ e de $\mathbf{n}$ são: o fato de serem abertas ou fechadas, o seu arranjo geométrico e a forma de suas paredes. A equação $\mathrm{P}$ se torna a equação $\mathrm{O}$ quando $\mathbf{C}=1$ e $\mathbf{n}=2$. Numa simulação com células abertas de uma esponja do tipo tetrakaidecaedral, Roberts e Carboczi [19] obtiveram os valores de $\mathbf{C}=0,93$ e $\mathbf{n}=2,04$, para $\rho / \rho_{0}<0,5$, muito próximos dos valores 1 e 2 , respectivamente.

Por outro lado, o valor de $\mathbf{k}$ para $\mu_{0}=0,31$ é 1,90 (Tabela I), transformando a equação $\mathrm{H}$ em:

$$
E(P)=E_{0} \cdot\left(1-1,9 \cdot P+0,9 \cdot P^{2}\right)
$$

que é a equação atribuída a Mackenzie, referenciada por Kingery [3].

Também com as mesmas hipóteses anteriores, Hashin [20], referenciado por Dörre e Hübner [16], propõe outra solução para o módulo de Young:

$$
E(P)=E_{0}\left[1-\frac{q \cdot P}{1+(q-1) \cdot P}\right]
$$

onde q é uma constante.

Os mesmos autores referenciam ainda Spriggs [21], que propõe uma expressão baseada no princípio da carga residual suportada pela secção transversal da matriz e na mínima distância entre os poros e que é dada por:

$$
E(P)=E_{0} \cdot \exp (-p \cdot P)
$$

onde p é uma constante. Knudsen [22] mostrou que a equação $\mathrm{S}$ apresenta bons resultados para a alumina na faixa de 
porosidade entre 0 e 0,40 , da qual deduziu um valor para $\mathbf{p}$ igual a 3,95. Outros valores encontrados para $\mathbf{p}$, no caso da alumina, são: 3,24 de Chung e Simmons [23]; 4,17 de Soga e Anderson [24], para porosidade até 0,05 ; e 3,5 $\pm 0,1$ de Rice [25] por compilação de dados.

Kingery [3] referencia um modelo de Voigt que calcula o módulo de Young para um material bifásico, no seu limite superior, em que a deformação sofrida por ambas as fases é a mesma. A expressão matemática decorrente desse modelo é dada por:

$$
E=V_{2} \cdot E_{2}+\left(1-V_{2}\right) \cdot E_{1}
$$

onde $\mathbf{V}_{2}$ é a fração volumétrica de uma fase 2 que tem seu módulo de Young igual a $\mathbf{E}_{\mathbf{2}}$ e $\left(1-\mathbf{V}_{2}\right)$ é a fração volumétrica da fase 1 que tem módulo $\mathbf{E}_{1}$. Adotando-se a porosidade como sendo a fase 2, cujo valor de $\mathbf{E}_{\mathbf{2}}$ deve-se atribuir o valor zero, e por definição $\mathbf{V}_{2}=\mathbf{P}$, a equação $\mathrm{T}$ se converte em

$$
E(P)=E_{0} \cdot(1-P)
$$

Cabe aqui destacar a importância de se ter um processo e um material ideal que pudesse servir para testar os diferentes modelos aqui apresentados. A principal característica desse processo seria permitir a obtenção do material com ampla variação de porosidade, mantendo as mesmas características macroestruturais.

\section{MATERIAIS E MÉTODOS}

Para a preparação das espumas cerâmicas efetuou-se o seguinte procedimento: preparou-se uma suspensão contendo $35 \%$ vol. de alumina calcinada (A-1000 SG, Alcoa, Brasil) dispersa em uma solução aquosa contendo $25 \%$ em peso de monômeros à base de acrilato de amônio e metileno-bisacrilamida (Ciba, Inglaterra). Esta foi dispersada com noliacrilato de amônio (Dispex A-40, $\bar{M}_{w}=10.000 \mathrm{~g} / \mathrm{Mol}$, Ciba, Brasil) e em seguida desaglomerada em moinho de bolas durante 20 minutos. Foram separadas alíquotas da suspensão com massa variando entre 100 e $520 \mathrm{~g}$, às quais adicionou-se catalisador (tetrametiletilenodiamina, Aldrich) até atingir uma concentração de $2 \mu \mathrm{L} / \mathrm{g}$ de suspensão. Adicionou-se ainda, a cada alíquota de suspensão, uma quantidade de um surfactante não-iônico (Lutensol ON-110, Basf, Brasil) cuja concentração diminuiu de $5,0 \cdot 10^{-3}$ para $1,1 \cdot 10^{-3} \mathrm{~g} / \mathrm{g}$ de suspensão, à medida que a quantidade de suspensão aumentou de 100 para $520 \mathrm{~g}$, de modo a proporcionar espumas com densidade variada após agitação. Cada alíquota de suspensão foi agitada em uma batedeira doméstica até a produção de um volume de aproximadamente $500 \mathrm{~mL}$ de espuma, após o que se adicionou $0,1 \mu \mathrm{L} / \mathrm{g}$ de suspensão de iniciador (solução aquosa 2:1 de persulfato de amônio, Aldrich). A composição e o procedimento empregados nesta etapa foram baseados em um estudo anterior que abordou a relação entre a concentração e o tipo de surfactante sobre variáveis tais como densidade e permeabilidade de corpos obtidos através desta técnica [26]. Após rápida homogeneização, a espuma foi vertida em um molde de alumínio com dimensões de $17,0 \times 9,1 \times 2,7 \mathrm{~cm}^{3}$, onde ocorreu a gelificação.

Os corpos obtidos foram retirados do molde, secos em temperatura ambiente durante 72 horas e em seguida sinterizados. Iniciou-se o aquecimento com uma taxa de aquecimento de $1{ }^{\circ} \mathrm{C} / \mathrm{min}$ até $350{ }^{\circ} \mathrm{C}$, mantendo esta temperatura por 1 hora para a eliminação do material orgânico. A seguir, com uma taxa de $5{ }^{\circ} \mathrm{C} / \mathrm{min}$ elevou-se a temperatura até $1650{ }^{\circ} \mathrm{C}$, que foi mantida por 3 horas. Seguiu-se o resfriamento a uma taxa de $10{ }^{\circ} \mathrm{C} / \mathrm{min}$.

Cada placa foi retificada e em seguida secionada de modo a se obter barras prismáticas, com dimensões de aproximadamente $14,0 \times 2,0 \times 0,75 \mathrm{~cm}^{3}$, adequadas para a medição dos módulos elásticos pela ressonância de barras.

A massa específica das esponjas foi caracterizada pelo método geométrico (massa/volume), com os mesmos corpos-de-prova utilizados para medir o módulo elástico. A porosidade total foi calculada com o auxílio da equação N. O erro experimental estimado na medida da densidade é de $0,2 \%$, ou seja, de $\pm 0,001 \mathrm{~g} / \mathrm{cm}^{3}$.

$\mathrm{Na}$ Tabela II mostram-se a densidade, a densidade relativa e a porosidade total para cada uma das amostras.

A descrição do equipamento utilizado, bem como do procedimento para a obtenção das propriedades elásticas, estão relatados em Paiva e Rodrigues [27].

Para cada uma das amostras foram realizadas as medidas das propriedades elásticas aplicando-se simultaneamente vibração mecânica de flexão e de torção à temperatura ambiente. Foi realizada uma varredura na freqüência de $1 \mathrm{kHz}$ a $40 \mathrm{kHz}$ nas amostras a fim de se obter os três primeiros picos de ressonância para cada um dos modos de vibração. A identificação desses picos foi realizada por um software desenvolvido em linguagem de programação Delphi. Esse programa controla o sistema e calcula em tempo real o módulo de cisalhamento, o módulo de Young e a razão de Poisson. Os três primeiros picos de ressonância de cada um dos modos de vibração são utilizados nos cálculos gerando os valores médios para aquelas propriedades.

Foram marcados nas amostras os pontos do apoio $(0,224 \times \mathbf{L}$ a partir de cada uma das extremidades $)$ e os pontos de contato do excitador e receptor $(0,3 \times \mathbf{L})$. As posições do excitador e do receptor são tais que permitem a vibração simultânea flexional e torcional.

Foram relacionados os três primeiros picos de ressonância por flexão e por torção por meio da verificação da razão entre suas freqüências. No caso da flexão, a razão teórica entre as freqüências do segundo $\left(\mathbf{f}_{\mathbf{f} 2}\right)$ e do primeiro pico $\left(\mathbf{f}_{\mathbf{f} 1}\right)$ e entre as do terceiro $\left(\mathbf{f}_{\mathbf{f z}}\right)$ e do primeiro pico $\left(\mathbf{f}_{\mathbf{f} 1}\right)$ são representadas por funções polinomiais, obtidas pela regressão de gráficos apresentados por Pickett [8] e pela norma ASTM C747 [28], que são expressas pelas seguintes equações: onde $\mathbf{r}$ é a razão entre o raio de giro e o comprimento da amostra. $\mathrm{O}$ raio de giro é definido pela raiz quadrada da razão 


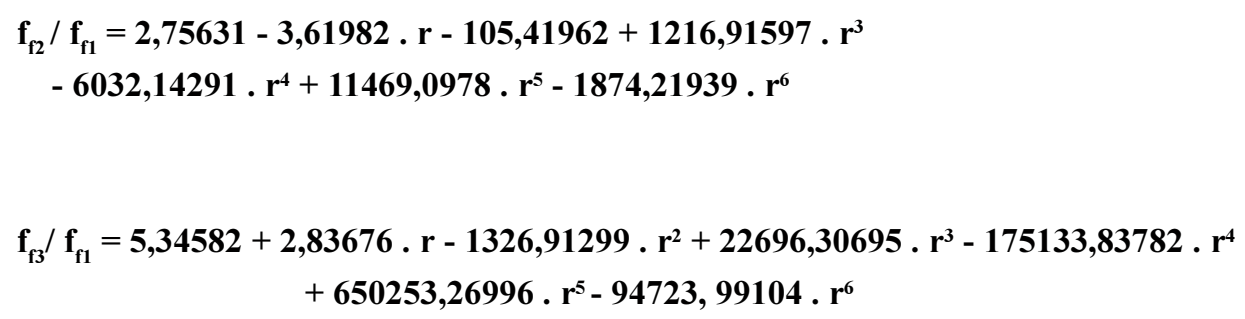

entre o momento de inércia e a área da secção transversal [29]. Para a vibração torcional, a razão entre as freqüências do segundo e do primeiro pico é aproximadamente 2 e entre as do terceiro e do primeiro pico é aproximadamente 3 , valores estes obtidos a partir da equação A. Para as dimensões dos corpos de prova deste trabalho, os erros envolvidos nessas aproximações são menores do que $0,3 \%$.

\section{RESULTADOS E DISCUSSÃO}

O módulo de Young, E, e o módulo de cisalhamento, $\mathbf{G}$, ambos medidos em função da porosidade total, na temperatura ambiente, são mostrados na Fig. 1. Pode-se observar que os módulos elásticos decrescem com o aumento da porosidade total.

Calculando-se simultaneamente os valores de $\mathbf{G}, \mathbf{E}$ e $\mu$, a razão de Poisson apresentou uma grande variação, desde 0,23 até 0,53 , com um valor médio de 0,35 e uma tendência de aumento com o aumento da porosidade, conforme mostrado na Fig. 2. Isso contraria artigos recentes de Asmani et al. [13] e Chang et al. [30], que correlacionaram a razão de Poisson com a porosidade em uma cerâmica à base de alumina e mostraram uma queda na razão de Poisson com o aumento da porosidade, na faixa de 0 a $25 \%$-vol. No entanto, a faixa de porosidade trabalhada por esses autores é bastante abaixo da faixa deste trabalho.

Por outro lado, o valor de $\mu=0,35$ é alto se considerado a

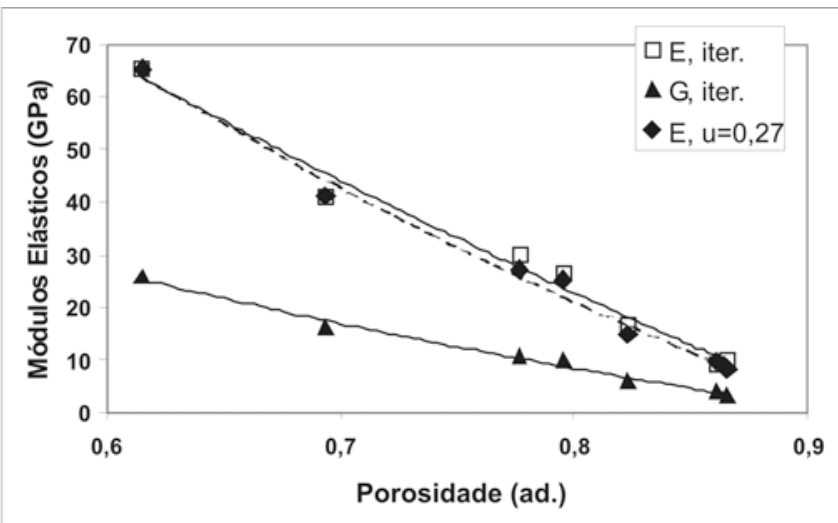

Figura 1: Influência da porosidade nas propriedades elásticas das esponjas de alumina à temperatura ambiente. Na legenda interna, "iter." significa iterativo e "u" é $\mu$. As linhas são apenas para indicar uma tendência.

[Figure 1: Influence of the porosity on the elastic properties of the alumina foams under room temperature. "iter." means iterative and " $u$ " is $\mu$. The lines are only to guide the eyes.] alumina densa, porém não se pode descartar uma influência da macroestrutura da espuma sobre essa propriedade, como mostrado no parágrafo anterior pelos trabalhos de Asmani et al. [13] e Chang et al. [30]. Adotando-se o valor indicado por Dörre e Hübner [16], de um trabalho de Cobles e Kingery para a razão de Poisson da alumina, $\mu=0,27$, a Fig. 1 mostra que o módulo de Young calculado com esse valor fixo de $\mu$, a

Tabela II - Valores da densidade geométrica e da porosidade total das 7 esponjas cerâmicas estudadas neste trabalho. $O$ valor de $\rho_{0}$ adotado foi de $3,95 \mathrm{~g} / \mathrm{cm}^{3}$.

[Table II - Geometrical density and total porosity of the 7 ceramic foams produced in this work $\left(\rho_{0}=3.95 \mathrm{~g} / \mathrm{cm}^{3}\right)$.]

\begin{tabular}{ccc}
\hline$\rho\left(\mathbf{g} / \mathbf{c m}^{3}\right)$ & $\rho / \rho_{\mathbf{0}}$ (ad.) & $\mathbf{P ( \% )}$ \\
\hline 0,531 & 0,134 & 86,6 \\
\hline 0,548 & 0,139 & 86,1 \\
\hline 0,698 & 0,177 & 82,3 \\
\hline 0,807 & 0,204 & 79,6 \\
\hline 0,882 & 0,223 & 77,7 \\
\hline 1,212 & 0,307 & 69,3 \\
\hline 1,520 & 0,385 & 61,5 \\
\hline
\end{tabular}

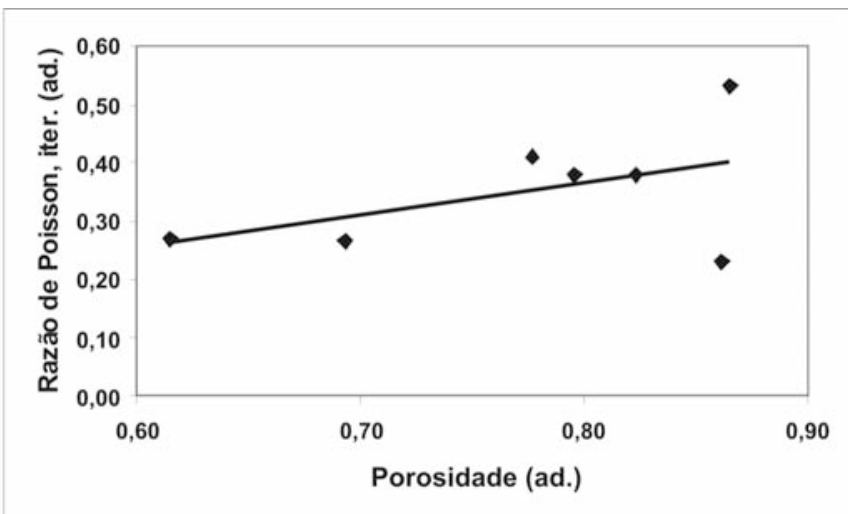

Figura 2: Razão de Poisson em função da porosidade total das esponjas calculada iterativamente a partir das freqüências de ressonância flexionais e torcionais. A linha é somente para indicar uma tendência.

[Figure 2: Poisson's ratio as a function of the foams total porosity. The Poisson ratio values were iteratively calculated from the flexural and torsional resonance frequencies. The lines are only to guide the eyes.] 
partir dos valores originais de $\mathbf{G}$ e empregando-se a equação $\mathbf{E}$, não difere muito dos resultados do processo iterativo. Isso revela que, no caso de esponjas cerâmicas de alta porosidade, o método iterativo para a determinação simultânea de $\mathbf{G}, \mathbf{E}$ e $\mu$ não é adequado para a determinação de $\mu$. Esse parâmetro parece ser extremamente sensível a algum efeito do processo de modo que grandes variações no seu valor praticamente não afetam os valores do módulo de Young.

Neste trabalho, decidiu-se usar os valores do módulo de Young obtidos com o valor fixo de $\mu=0,27$.

A Fig. 3 mostra a variação do módulo de Young relativo em função da porosidade total segundo os dados experimentais (pontos), além das curvas correspondentes aos diversos modelos apresentados neste artigo, a saber: Boccaccini [14], equações $F$ e G, tanto para poros esféricos $(\mathbf{z} / \mathbf{x}=1,0 ; \mathbf{s}=1,21)$ como para poros cilíndricos de orientação aleatória $(\mathbf{z} / \mathbf{x}=1,5 ; \mathbf{s}=1,25)$; Mackenzie, equação Q, referenciada por Kingery [3], que é decorrente das equações H e L para $\mu=0,31$ e k=1,90; Mackenzie, equação $M$, referenciada por Gibson e Ashby [18], que é decorrente do valor de $\mu=0,20$ e $\mathbf{k}=2,00$; Voigt, referenciado por Kingery [3], equação U; Hashin [20], referenciado por Dörre e Hübner [16], equação R, com o valor de $\mathbf{q}=3,24$; e finalmente Spriggs [21], também referenciado por

Dörre e Hübner [16], equação $S$, com valor de $\mathbf{p}=4,0$.

Pode-se observar que os resultados experimentais estão muito próximos das curvas traçadas segundo o modelo de Mackenzie, entre as linhas definidas $\operatorname{com} \mu=0,20(\mathbf{k}=2,00)$, como adotado por Gibson e Ashby [18], e com $\mu=0,31(\mathbf{k}=1,90)$, como exposto por Kingery [3], sendo que esse valor de $\mu$ pode ser considerado acima dos valores comuns para materiais cerâmicos.

A Fig. 4 mostra como correções nos valores dos parâmetros que controlam os modelos da relação $\mathbf{E} \times \mathbf{P}$ podem fazer com que se consiga boa concordância entre estes e os pontos experimentais. Nota-se que, na faixa de porosidade do material estudado neste trabalho, ajustes muito bons são conseguidos com o modelo de

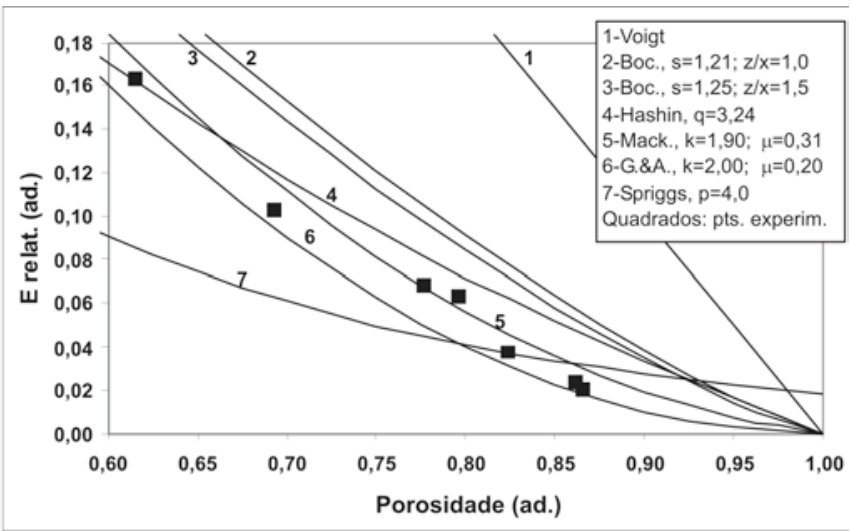

Figura 3: Influência da porosidade no módulo de Young relativo segundo os diversos modelos matemáticos (curvas contínuas) apresentados neste trabalho e os resultados experimentais deste artigo (pontos). "G.\&A." significa Gibson e Ashby.

[Figure 3: Influence of the porosity on the relative Young's modulus after different mathematical models (continuous lines) presented in the text. It is also plotted the experimental results of this paper (points). "G.\&A." means Gibson and Ashby.]
Boccaccini para $\mathbf{z} / \mathbf{x}=3,1$ e $\mathbf{s}=1,48$ e também com o modelo de Mackenzie para $\mathbf{k}=1,94$ e $\mu=0,27$. Portanto, para o valor adotado neste trabalho para a razão de Poisson, o ajuste pelo modelo de Mackenzie pode ser considerado muito bom.

Os modelos que permitem a adequação da morfologia dos poros no valor de $\mathbf{E}$ parecem ser, em princípio, os mais adequados. Por outro lado seria necessário considerar-se o limite de aplicação destas equações, pois no final não se sabe se a equação está correta ou se o bom ajuste é puramente matemático. Os pontos experimentais determinados nesta pesquisa são bem representados pelo modelo de Boccaccini

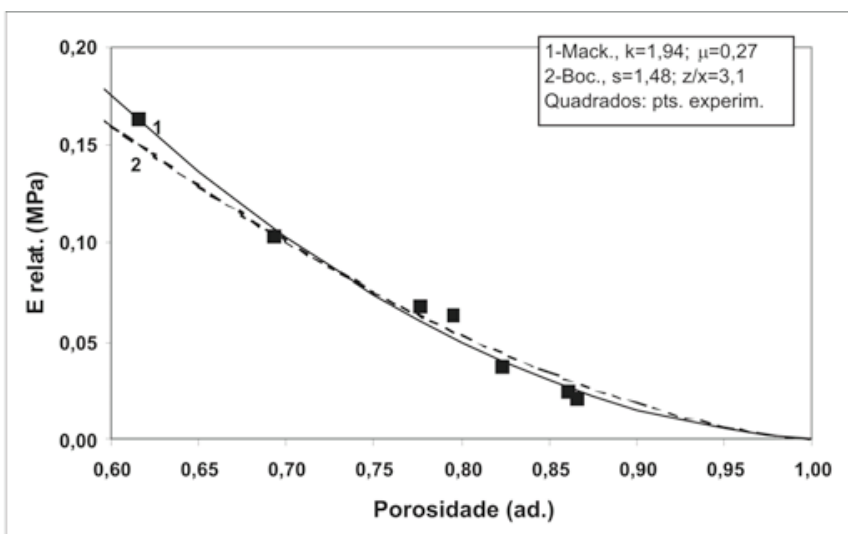

Figura 4: E x P experimental (pontos) comparado com alguns dos modelos tratados neste trabalho com parâmetros ajustados para uma boa regressão (curvas). Boccaccini, $\operatorname{com} \mathbf{z} / \mathbf{x}=3,1$ e $\mathbf{s}=1,48$; Mackenzie $\operatorname{com} \mathbf{k}=1,94$, que corresponde a $\mu_{0}=0,27$.

[Figure 4: Experimental $\boldsymbol{E} \times \boldsymbol{P}$ plot (points) compared to some of the models presented in this work. Fitting parameters applied in order to obtain a better regression (continuous lines): Boccaccini, with $z \boldsymbol{x}=3.1$ and $\boldsymbol{s}=1.48 ;$ Mackenzie, with $\boldsymbol{k}=1.94$, which corresponds to $\mu_{0}=0.27$.]

com os parâmetros $\mathbf{z} / \mathbf{x}=3,1$ e $\mathbf{s}=1,48$, conforme mostrado na Fig. 4. Isso sugere poros alongados e tal consideração é pertinente uma vez que há uma efetiva conexão entre os poros das esponjas, conforme observado na Fig. 5, que ilustra a macroestrutura celular desses materiais. Uma fileira de poros, mesmo que basicamente esféricos, porém interconectados, pode ser abstraída por um poro cilíndrico. No entanto, na macroestrutura mostrada na Fig. 5, pode-se ver que a interconectividade é tridimensional, muito mais complexa do que simples poros alongados.

Nesse contexto, o modelo de Mackenzie tem o mérito de depender apenas de um único parâmetro, que inclusive não é microestrutural, (k ou $\mu$ ) com o qual o ajuste da curva teórica aos pontos experimentais (Fig. 4) exige um valor para a razão de Poisson que tem um sentido físico claro e de valor numérico igual ao aceito para a alumina densa. No entanto, esse modelo foi deduzido considerando-se apenas poros esféricos fechados e uma porosidade de no máximo $50 \%$-vol.

A Fig. 6, mostra os valores experimentais (pontos) de $\mathbf{E}$ e de $\mathbf{G}$, em função da densidade relativa das esponjas cerâmicas. Essa figura mostra também as curvas teóricas que descrevem 


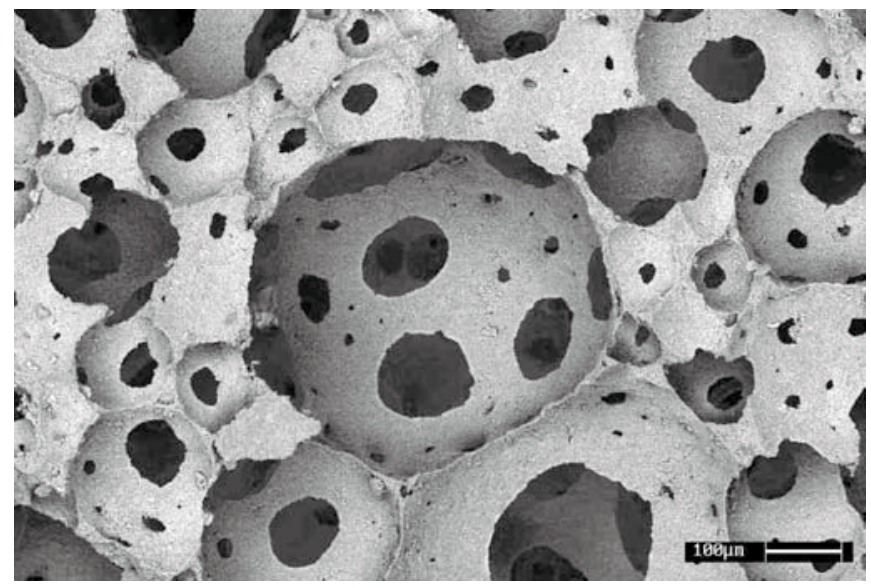

Figura 5: Fotografia da macroestrutura das esponjas de alumina preparadas pelo método de gelcasting. Os poros são basicamente esféricos e interconectados tridimensionalmente.

[Figure 5: SEM photo of the macrostructure of the alumina foams prepared via gelcasting. The pores are basically spherical and tridimensionally interconnected.]

o efeito da porosidade sobre estes dois parâmetros. Para todas as curvas calculadas dessa figura, o valor de $\mathbf{E}_{\mathbf{0}}$, adotado para a alumina, foi de $400 \mathrm{GPa}$.

Verifica-se, na Fig. 6, que a diminuição da densidade relativa (aumento da porosidade total) provocou uma redução de ambos os módulos elásticos, conforme previsto pelos modelos teóricos. Os valores do módulo de Young obtidos experimentalmente apresentaram boa concordância com os valores previstos pelo modelo de Mackenzie $(\mathbf{k}=1,9)$. Ver a linha contínua.

O modelo de Gibson e Ashby, por sua vez, é válido para células com geometria equiaxial. Ver a linha tracejada para $\mathbf{E}(\mathbf{k}=2,0$, com $\mathbf{C}=1$ e $\mathbf{n}=2)$ e a linha pontilhada para $\mathbf{G}\left(\mathbf{C}^{\prime}=3 / 8=0,375\right)$. Embora os poros presentes nas espumas

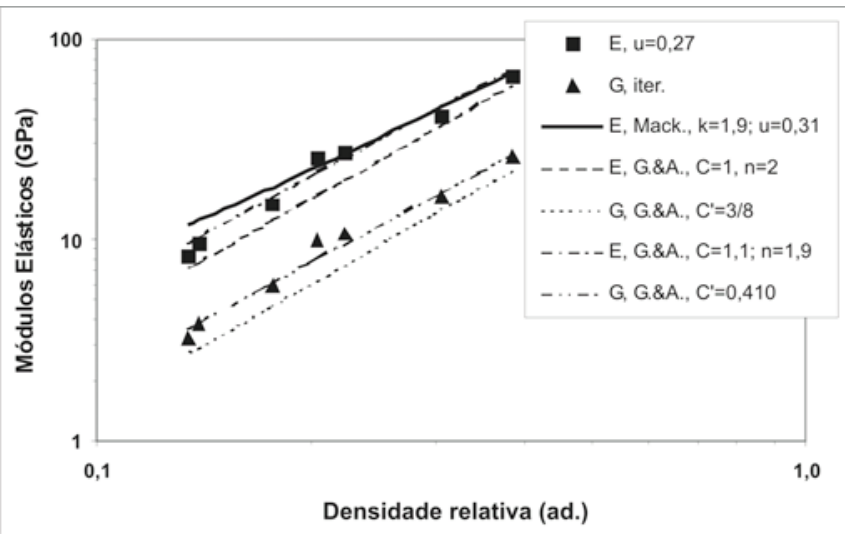

Figura 6: Módulos de Young, E, e de cisalhamento, G, versus a densidade relativa das esponjas de alumina. As linhas representam alguns modelos apresentados neste trabalho e os pontos são os valores experimentais. "G.\&A." significa Gibson e Ashby e "u" é $\mu$.

[Figure 6: Young's modulus, E, and shear modulus, G, as a function of the relative density of the alumina foams (points). The lines represent some of the models presented in this work. "G.\&A." means Gibson and Ashby and " $u$ " is $\mu$.] estudadas apresentem uma geometria aproximadamente equiaxial, desvios em relação à esfericidade podem ocorrer, como constatado em espumas de alumínio produzidas a partir do metal líquido [31]. O desvio entre o modelo de Gibson e Ashby e os dados experimentais pode estar relacionado, também, à origem empírica do valor sugerido para a constante $\mathbf{C}$ da equação P. Tentando-se um melhor ajuste para $\mathbf{E}$ e $\mathbf{G}$ usando o modelo de Gibson e Ashby, a linha traço-e-ponto é obtida com $\mathbf{C}=1,1$ e $\mathbf{n}=1,9$ e a linha traço-e-dois-pontos com $\mathbf{C}^{\prime}=0,410$.

\section{CONCLUSÕES}

Os valores experimentais do módulo de Young, determinados pela técnica de ressonância mecânica de barra, em função da porosidade das esponjas obtidas pelo método de gelcasting, são bem ajustados pelos modelos de Boccaccini com os parâmetros $\mathbf{s}=1,48$ e $\mathbf{z} / \mathbf{x}=3,1$ e de Mackenzie com os parâmetros $\mathbf{k}=1,94$ e $\mu=0,27$.

Embora o modelo de Boccaccini possibilite um bom ajuste dos pontos experimentais do módulo de Young em função da porosidade das esponjas, fica difícil compreender o significado físico do parâmetro $\mathbf{z} / \mathbf{x}=3,1$, poros alongados, sabendo que o material é composto de uma macroestrutura de células basicamente esféricas e interconectadas tridimensionalmente.

É um mérito do modelo de Mackenzie, com o parâmetro $\mathbf{k}=1,94$, associado ao valor 0,27 para a razão de Poisson, ter possibilitado excelente ajuste dos pontos experimentais do módulo de Young em função da porosidade das esponjas, mesmo sendo o modelo conseqüência da hipótese de poros esféricos fechados.

Embora o modelo de Gibson e Ashby seja recomendado especificamente para materiais celulares com células equiaxiais, este não se ajusta adequadamente aos valores experimentais do módulo de Young encontrados neste trabalho. O modelo de Gibson e Ashby, usualmente considerando a densidade relativa das esponjas, quando convertido em função da porosidade, coincide com o modelo de Mackenzie para os parâmetros $\mathbf{k}=2,0$ e $\mu=0,20$.

\section{AGRADECIMENTOS}

Os autores J. A. Rodrigues e V. C. Pandolfelli agradecem ao apoio dado pela FAPESP (Processos 97/01114-5 e 01/ 04324-8) e pelo CNPq; F. S. Ortega agradece a Capes; e A. E. M. Paiva agradece ao PICDT/Capes.

\section{REFERÊNCIAS}

[1] F. S. Ortega, A. E. M. Paiva, J. A. Rodrigues, V. C. Pandolfelli, Anais do $46^{\circ}$ Congresso Brasileiro de Cerâmica, S. Paulo, SP (2002), CD-ROM, p. 1200-1211.

[2] I. Nettleship, Key Eng. Mater. 122-124 (1996) 305-324.

[3] W. D. Kingery, H. K. Bowen, D. R. Uhlmann, Introduction to Ceramics, $2^{\text {nd }}$ ed., John Wiley $\&$ Sons, New York (1976) p. 1032.

[4] P. A. Sepulveda, Am. Ceram. Soc. Bull. 76, 10 (1997) 61-65. 
[5] E. Schreiber, A. L. Orson, N. Soga, Capítulo 4, in Elastic Constants and Their Measurement, McGraw-Hill (1973) 82-125. [6] American Society for Testings and Materials, C 885-87 (1987) 278-283.

[7] J. R. Nicholls, C. Mendes, P. Handboock, Materials at High Temperatures 12,

2,3 (1994) 85-94.

[8] G. Pickett, ASTM Proceedings, 45 (1945) 846-865.

[9] T. Kaneko, J. Non-Crystalline Solids 21 (1976) 435-439.

[10] R. M. Davies, Philosophical Magazine 25 (1938) 364-386.

[11] W. E. Tefft, S. Spinner, J. Res. Nat. Bur. Standards 65A (1961) 167-171.

[12] American Society for Testing and Materials, C 1198-96 (1996) 1-8.

[13] M. Asmani, C. Kermel, A. Leriche, M. Ourak, J. Eur. Ceram. Soc. 21 (2001) 1081-1086.

[14] D. N. Boccaccini, A. R. Boccaccini, J. Nondestructive Evaluation 16, 4 (1997) 187-192.

[15] G. Ondracek, Reviews on Powder Metallurgy and Physical Ceramics 3, 3-4 (1987) 205-322.

[16] E. Dörre, H. Hübner, Alumina Processing, Properties and Applications, Springer-Verlag, Berlin (1984) 74-104.

[17] J. K. Mackenzie, Proc. Phys. Soc. 63B (1950) 2-11.

[18] L. J. Gibson, F. M. Ashby, Cellular Solids - Structure and Properties, $2^{\text {nd }}$ ed., Cambridge University Press (1997) 175-234. [19] A. P. Roberts, E. J. Garboczi, Acta Mater. 49 (2001) 189197.
[20] Z. Hashin, Elasticity of ceramic systems, in R. M. Fulrath, J. A. Pask (Eds.), Ceramic microstructures, John Wiley and Sons, New York (1968) p. 313.

[21] R. M. Spriggs, J. Am. Ceram. Soc. 44 (1961) 628-629.

[22] F. P. Knudsen, J. Am. Ceram. Soc. 45 (1962) 94-95.

[23] D. H. Chung, G. Simmons, J. Appl. Phys. 39 (1968) 53165326.

[24] N. Soga, O. L. Anderson, J. Am. Ceram. Soc. 49 (1966) 318-322.

[25] R. W. Rice, Microstructure dependence of mechanical behavior of ceramics, in R. K. MacCrone (Ed.), Treatise on materials science and technology, Vol. 11, Properties and microstructure, Academic Press, New York (1977) p. 199-381. [26] F. S. Ortega, P. Sepulveda, M. D. M. Innocentini, V. C. Pandolfelli, Am. Ceram. Soc. Bull. 80, 4 (2001) 37-42.

[27] A. E. M. Paiva, J. A. Rodrigues, Anais do $45^{\circ}$ Congresso Brasileiro de Cerâmica, Florianópolis, SC, CDROM (2001) p. 2200301-2200313.

[28] American Society for Testing and Materials, C747-93 (1993) 1-8.

[29]A. C. Ugural, Mechanics of Materials, McGraw-Hill Inter. Ed., New York (1991) p. 393-395.

[30] L. S. Chang, T. H. Chuang, W. J. Wei, Materials Characterization 45 (2000) 221-226.

[31] A. E. Simone, L. J. Gibson, Acta Mater. 46, 9 (1998) 3109-3123.

(Rec. 11/12/03, Ac. 05/03/04) 